\title{
AProvechamiento DE LA ENERGÍA UNDIMOTRIZ EN EL ECUADOR
}

\section{ENERGY HARNESSING WAVE IN ECUADOR}

\author{
Ronald Rodríguez-Santos ${ }^{1, *}$, Manuel Chimbo-Campuzano ${ }^{2}$
}

\section{Resumen}

Un problema en el Ecuador años atrás era la dependencia de energías fósiles no renovables como el petróleo, recurso que es agotable y su costo varía según la Organización de Países Exportadores de Petróleo (OPEP). Con el cambio de la matriz energética y la gran inversión de nuestro país en energías renovables, se ha logrado actualmente tener una producción de energía renovable correspondiente al $51,78 \%$, lo cual representa 13.638,89 (GWh), fuente tomada del ARCONEL (Agencia de regulación y control de Electricidad). Aprovechando los recursos naturales tales como hídricos, solares, eólicos entre otros, desechando en parte gran producción de energía contaminante. Otra energía alternativa no explotada y en la que nos vamos a enfocar en el ensayo es la energía undimotriz, que consiste en la energía que llevan las olas a lo largo de los océanos del mundo, esta energía se genera a partir de la acción del viento en la superficie del mar. Con los datos obtenidos del Inocar se realizaron los cálculos necesarios para obtener el potencial energético de nuestros mares. Alcanzando un potencial energético muy bueno como se detalla en el artículo.

Palabras clave: energía mecánica, energía undimotriz, energía eléctrica, energía renovable y olas ecuatorianas.

\section{Abstract}

A problem in Ecuador years ago was dependence on energies fossils non-renewable as the petroleum. Resource that is exhaustible and its cost varies according to the OPEP (Organization of Petroleum Exporting Countries). With the change of the energy matrix and the great investment of our country in renewable energies, has been achieved at present have a production renewable energy corresponding to $51.78 \%$, which represents $13,638.89$ (GWh), source taken from the ARCONEL (Agency of regulation and control of Electricity). Leveraging natural resources such as: hydric, solar, eolic, among others, discarding in part large production of polluting energy. Another unexploded alternative energy in which are going to focus in the test is the wave energy, which consists of the energy carried by the waves along the world's oceans; this energy is generated from the action of the wind on the surface of the sea. With the data obtained from INOCAR, were performed calculations required to obtain the energy potential of our seas. Reaching an energy potential very good as detailed in the article.

Keywords: Energy Mechanical, Energy Wave, Energy Electrical, Renewable Energy and Ecuadorian Waves.

\footnotetext{
${ }^{1, *}$ Carrera de Ingeniería Eléctrica, Universidad Politécnica Salesiana, sede Cuenca - Ecuador, Autor para correspondencia : rrodriguezs1@est.ups.edu.ec

${ }^{2}$ Carrera de Ingeniería Eléctrica, Universidad Politécnica Salesiana, sede Cuenca - Ecuador.
}

Recibido: 29-09-2016, aprobado tras revisión: 16-12-2016

Forma sugerida de citación: Rodríguez, R.; Chimbo, M. (2017). «Aprovechamiento de la energía undimotriz en el Ecuador». InGENIUS. N. ${ }^{\circ} 17$, (Enero-Junio). pp. 23-28. ISSN: 1390-650X. 


\section{Introducción}

La contaminación del medioambiente es sin lugar a duda el principal motivo por el cual obliga buscar mecanismos o métodos para la producción de energía eléctrica de una forma no agresiva para el medioambiente. Actualmente no existen suficientes estudios que indiquen cuáles son los potenciales energéticos que se puede aprovechar en el mar. Es por eso por lo que en el siguiente artículo se realizará un estudio del potencial energético de la energía undimotriz en el Ecuador [1,2].

En el transcurso de los años ha existido un gran interés en sistemas para aprovechar la energía undimotriz en el mundo. En el Ecuador aún no se ha aplicado a gran escala la generación de electricidad a través de este tipo de energía, debido a la falta de interés de las entidades públicas.

Para poder generar energía eléctrica a través del movimiento de las olas, se debe instrumentar estudios sobre los oleajes en las costas ecuatorianas; nos podemos ayudar con información de instituciones de control marítimo como el Instituto Geofísico de la Armada, Inocar, con esos datos obtenidos realizaremos los cálculos aproximados para obtener la energía, longitud de la ola y la potencia por metro de frente de ola de nuestros mares. Según lo calculado se analizará si es factible su implementación $[1,2]$.

Con respecto a los estudios realizados se conoce que el oleaje que arriba a las costas del Ecuador tiene un promedio de olas de $\mathbf{2} \mathbf{~ m}$, en profundidades de hasta $\mathbf{2 0} \mathbf{m}$ y de $\mathbf{2 , 5} \mathbf{m}$ en profundidades superiores, sin embargo, estos valores son solo referenciales. Pueden existir cambios de acuerdo con los fenómenos climáticos existentes en el país [1].

Las olas de los mares y océanos son un derivado terciario de la energía solar, pues la radiación solar incide sobre la superficie de la tierra y provoca un calentamiento desigual de la misma, produciendo en la atmósfera zonas con distinta presión. Esto produce desplazamientos de aire de uno a otro lugar, es decir, generando vientos [1].

Son estos vientos, los que al desplazarse sobre la superficie del mar llevan a cabo el rozamiento de las moléculas de aire con el agua, transfiriendo a esta parte de su energía y generando las olas, estas actúan como un acumulador de energía pues la almacenan y la transportan de un lugar a otro con poquísimas pérdidas, lo que da lugar a que la energía de las olas que se producen en cualquier parte del océano acabe en las costas, así el 0,3\% de la energía solar que llega a la superficie terrestre se transforma en energía undimotriz [1].

\section{Características de la energía undi- motriz}

La energía undimotriz nos permite obtener energía eléctrica (electricidad) a partir de la energía mecánica generada por el movimiento o golpe de las olas. Es una de las energías renovables con más relevancia en los últimos años. Para generar energía eléctrica a través del movimiento de las olas, se tiene que realizar estudios acerca de los oleajes en la zona costera del país. Se deben establecer programas de monitoreo ubicados en áreas claves y de preferencia en 50 a $100 \mathrm{~m}$ de profundidad y alejados a unos dos kilómetros del perfil costanero ecuatoriano. El estudio se lo realizará en las costas del Ecuador, véase la Figura 1 [1-3].

\subsection{Mar ecuatoriano, potencial energético}

El estudio sobre las características de las olas se fundamenta en la información adquirida de la base de datos disponibles del Inocar. En la Figura 2, se muestra el área correspondiente al perfil costanero donde se analiza la potencia en $K W / m$ en el Ecuador. El Inocar es la entidad que realiza el registro del comportamiento del mar. Se aprecia un contenido energético del oleaje en el territorio ecuatoriano, el cual tiene un valor de $14 \mathrm{KW} / \mathrm{m}$ promedio de potencia $[3,4]$.

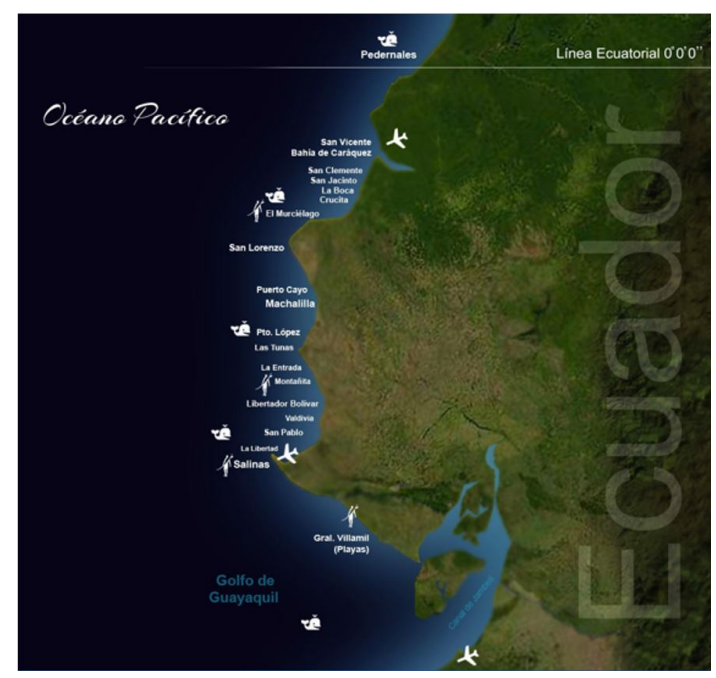

Figura 1. Zona costera del Ecuador. [3]

Las condiciones de las olas en aguas profundas a más de $2000 m$ se mantienen constantes. Para un análisis del potencial energético se debe averiguar la frecuencia y longitud de la ola en el mar. Para los sistemas de detección y monitoreo se emplean boyas. Esta boya se encuentra ubicada al suroeste de la ciudad de Esmeraldas para la detección de periodos de aguajes. Según el último informe, las olas tienen una altura media de 1,5 a $2 \mathrm{~m}$. Y el periodo de las olas está comprendido entre 12 a $15 s[3,5,9]$. 


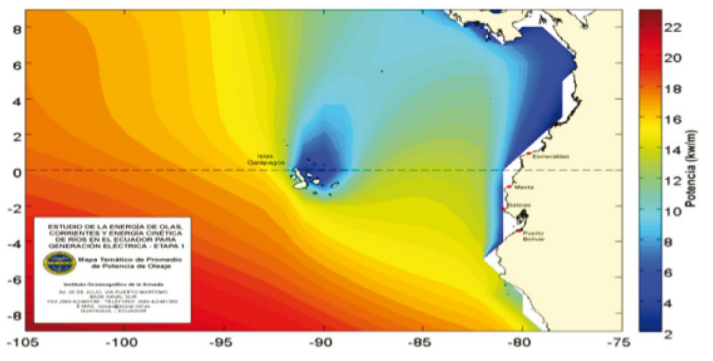

Figura 2. Área de estudios marinos para determinar el potencial energético. [3]

A continuación, en la Figura 3 se observa una grilla del Ecuador, donde se muestra en metros los oleajes en el perfil costanero.

En la Figura 4 se muestra las velocidades de las olas en la costa ecuatoriana que no superan el $1 \mathrm{~m} / \mathrm{s}$. A partir de la gráfica se llega a la conclusión de que las zonas con mayor velocidad están en las provincias del Guayas y El Oro con registros máximos de 3 a $4 \mathrm{~m} / \mathrm{s}$ [3].

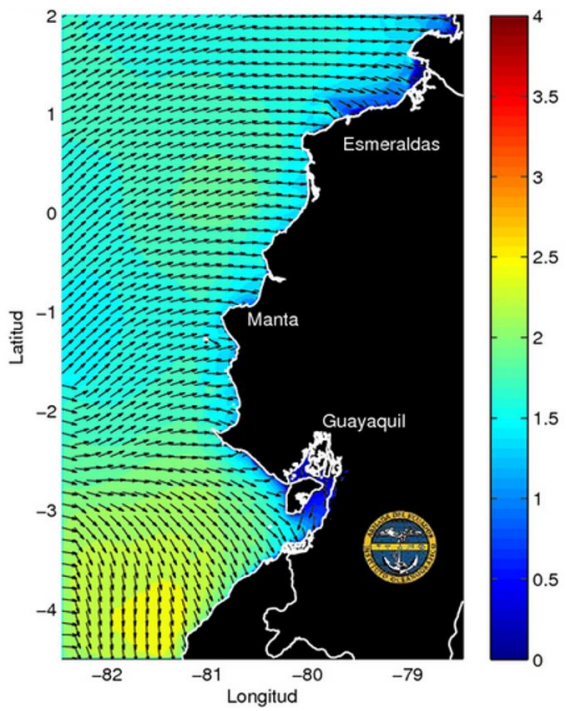

Figura 3. Modelo de operación Inocar-SWAM altura de oleaje. [3]

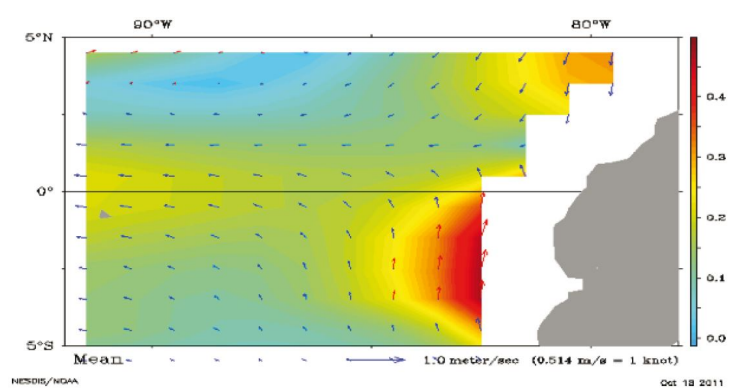

Figura 4. Velocidades promedio de las olas en la costa ecuatoriana. [3]
2.2. Ventajas y desventajas de la energía undimotriz

\section{Ventajas}

- Permite usos alternativos a la energía eléctrica, como la producción de hidrógeno o la obtención de agua potable del mar [6].

- Se trata de un recurso concentrado, predecible, cercano a grandes consumidores y de alta disponibilidad [6].

- Las olas pueden desplazarse durante largas distancias sin perder energía [6].

- Ofrece alta disponibilidad ya que es un recurso abundante y con flujos de energía elevados [6].

- Algunos dispositivos permiten el uso de generadores síncronos, que participarían en el control de la potencia reactiva de la red [6].

- El desarrollo de esta energía dará seguridad de suministro energético a regiones remotas, igual que ocurre con otras energías renovables [6].

- Se impulsará la diversificación de empleo y estimulará pymes e industrias en declive como las de construcción de barcos [6].

- Es una energía limpia, no emite gases contaminantes a la atmósfera [6].

\section{Desventajas}

- Varios dispositivos han fracasado ante tormentas, pues la carga que debe soportar el dispositivo en las condiciones más adversas (por ejemplo huracanes) puede ser 100 veces mayor que su carga nominal. Asimismo, aunque las condiciones sean mejores, los dispositivos tienen que soportar la energía cinética de las olas ininterrumpidamente [6].

- Los dispositivos alejados de la costa tienen unos costes importantes, debidos no solo al mantenimiento y a la instalación, sino también al sistema de amarre, que debe ser revisado y sustituido cada poco tiempo, garantizando que ninguna pieza se pierda y quede a la deriva [6].

- La vegetación marina crece en todo lo que se instala en el mar [6].

- El efecto corrosivo del agua salada tiene consecuencias muy negativas en distintos materiales. Por ejemplo, provoca que el acero se oxide, que el cobre se disuelva y que el aluminio desaparezca $[6]$. 
- El diseño es complejo. Como afirma Creus Solé: «lo que se diseña en la bañera no funciona en el $\operatorname{mar} \gg[6]$.

- La irregularidad en la amplitud, en la fase y en la dirección de la ola (la potencia entrante es aleatoria) hace difícil que un dispositivo obtenga el máximo rendimiento en todo el intervalo de frecuencias de excitación [6].

- En general, la eficiencia de la conversión energética no es alta [6].

- El coste de la planta de conversión es muy alto. Pese a que el coste de la materia prima es nulo (no hay que olvidar que el fluido de trabajo es el agua o el aire), el coste de construcción, que implica los sistemas de anclaje, los cierres herméticos, los cojinetes, etc., es muy elevado [6].

Todas estas características parecen manifestar que el diseño de un dispositivo de energía undimotriz debe ser, desde el punto de vista de aprovechamiento energético, sofisticado y fiable y, por otro lado, económicamente viable; aunque como citaba el autor de Energy from Waves, David Michael Ross: «La energía de las olas no fue diseñada para ahorrar dinero, sino para salvar el mundo» [6].

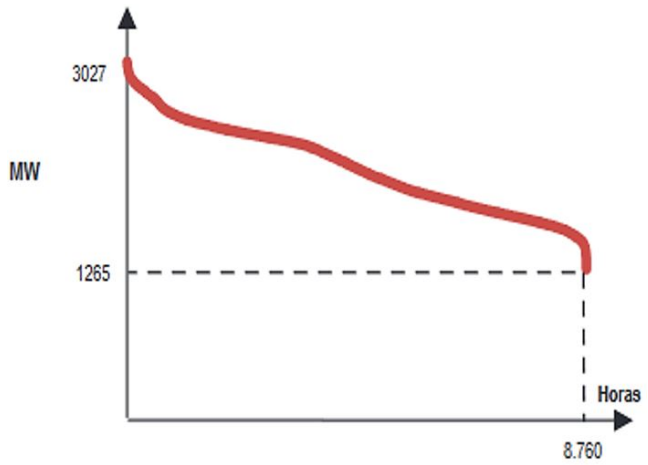

Figura 5. Demanda de energía eléctrica en el Ecuador. [12]

\subsection{Potencial energético de la energía undi- motriz en el Ecuador}

Según la Unesco con un boletín emitido en el año 2009 la cantidad de energía undimotriz disponible en todo nuestro planeta está en el orden de los $200 G W$ [7]. El consumo de energía en nuestro país según el Arconel se muestra en la Figura 5. Se observa las demandas máximas que ocurren en las horas picos [12]. Haciendo un promedio de consumo mínimo y máximo obtenemos una demanda promedio de energía en nuestro país de $2146 M W h$.

El mapa global de energía undimotriz en forma de potencia por unidad lineal se puede observar en la
Figura 6, apreciando que la potencia latente en nuestro litoral marítimo es aproximadamente de $20 \mathrm{KW}$ por cada metro de ola.

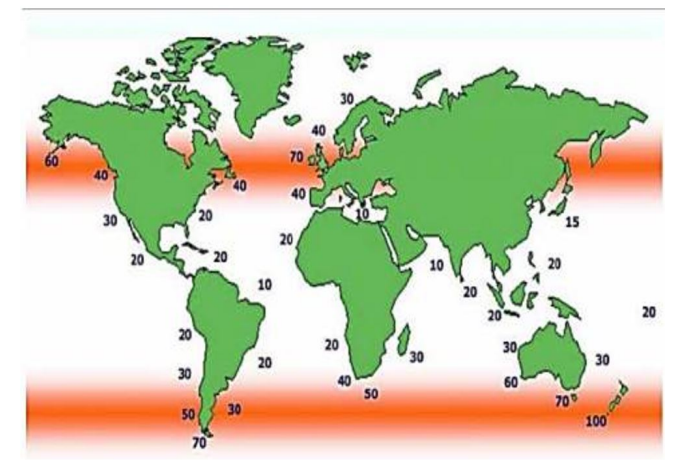

Figura 6. Niveles de energía de las olas en el mundo. [8]

El viento, al rozar con la superficie del mar, transmite su energía al agua y provoca movimientos circulares que se van desplazando. Existen dos maneras de extraer esta energía, través de la energía de las mareas (mareomotriz) y la energía de las olas (undimotriz) [8]. La ola del mar se puede considerar una onda sinusoidal como se puede observar en la Figura 7.

La energía cinética de esta ola con respecto a su nivel basal que es el punto más profundo en el que se percibe el movimiento de las olas [1-10]; está dada por la ecuación 1:

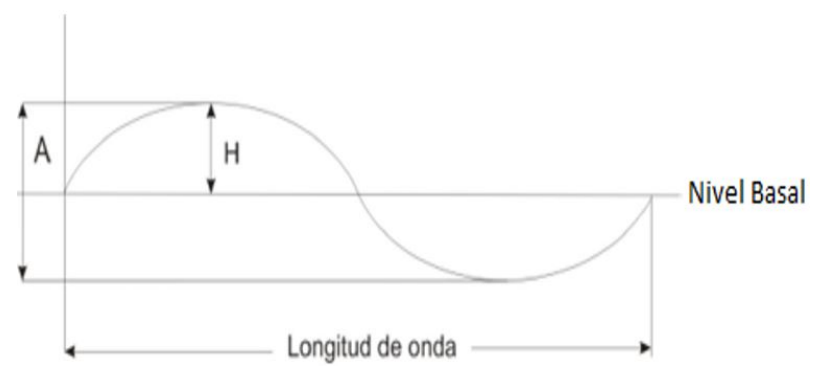

Figura 7. Parámetros de una ola sinusoidal. [11]

$$
E_{c}=\frac{1}{2} m v^{2}
$$

Donde $(\mathrm{m})$ es la masa de agua y (v) la velocidad de la ola, estas vienen dadas por las ecuaciones 2,3 .

$$
\begin{gathered}
m=\rho \times \frac{H}{2} \times \frac{\lambda}{2} b \\
v=\sqrt{\frac{g \lambda}{2 \pi}}
\end{gathered}
$$

Para calcular la longitud de onda debemos aplicar la ecuación 4:

$$
\lambda=\sqrt{g H} T
$$

Donde

$\rho$ : Densidad de agua, $\lambda$ : Longitud de onda, $b$ : Ancho de frente de onda, $A$ : Amplitud de onda, 
$H$ : Altura de ola, $g$ : Gravedad, $T$ : Periodo.

Estos parámetros de mares ecuatorianos los conseguimos de la página web del Instituto Oceanográfico de la Armada, Inocar, para así poder calcular la cantidad de energía potencial y cinética por metro de frente de onda que produciría la ola ecuatoriana [5]. Tanto la altura de la ola ecuatoriana como los otros parámetros dependerán mucho de las estaciones climáticas, pero aproximadamente tienen una altura de 1,5 a $2 \mathrm{~m}$; sacaremos un promedio de estas alturas para obtener una altura de $1,75 \mathrm{~m}$, mientras que el periodo estará entre 12 y $18 s$, haciendo el mismo procedimiento anterior conseguimos un periodo promedio de $15 s \mathrm{y}$ la gravedad será $9,81 \mathrm{~m} / \mathrm{s}^{2}$.

Con estos parámetros podremos calcular la longitud de la onda utilizando la ecuación 4:

$$
\lambda=\sqrt{1,75 \times 9,81} \times 15=62,15 \mathrm{~m}
$$

Con este valor utilizaremos la ecuación 3 para calcular la velocidad de la onda:

$$
v=\sqrt{\frac{9,81 \times 62,15}{2 \pi}}=9,85 \frac{\mathrm{m}}{\mathrm{s}}
$$

Ahora se calculará la masa del agua que depende de la densidad del agua, que es igual a $1027 \mathrm{~kg} / \mathrm{m}^{3}$ según el Instituto Nacional de Preinversión INP y el ancho de frente de onda (b) será de $1 \mathrm{~m}$, a partir de estos datos se hará el cálculo de potencia por metro de frente de ola.

$$
m=1027 \times \frac{1,75}{2} \times \frac{62,15}{2} 1=27870,6 \mathrm{~kg}
$$

Con los valores previamente calculados, se obtiene ahora el valor de la energía cinética de la ola ecuatoriana utilizando la ecuación 1 :

$$
E_{c}=\frac{1}{2} \times 27870,6 \mathrm{~kg} \times\left(9,85 \frac{\mathrm{m}}{\mathrm{s}}\right)^{2}=1,354 \mathrm{MJ}
$$

Para la potencia por metro de frente de ola se calcula utilizando la ecuación 9.

$$
\begin{gathered}
P=\frac{E_{c}}{T} \\
P=\frac{1,254 \mathrm{MJ}}{15 \mathrm{seg}}=90,311 \frac{\mathrm{kW}}{\mathrm{mt} \text { de frente de ola }}
\end{gathered}
$$

\section{Sistemas de extracción de la energía undimotriz}

A partir de los resultados obtenidos de la potencia que ofrece en las costas ecuatorianas se plantean los siguientes generadores para poder captar esa energía y convertirla en energía eléctrica. La tecnología para poner en funcionamiento sistemas de extracción de energía undimotriz es costosa debido que para instalar los generadores y equipos se deben hacer a gran distancia con respecto a la costa. Además, la construcción de los equipos deben ser materiales lo bastante resistentes y anticorrosivos para el agua salada. Pero a partir de nuestro análisis de la potencia del golpe de ola vemos que estos generadores son muy grandes para nuestra costa por lo que se plantea un nuevo generador, pero quedaría para futuros análisis.

\subsection{Generadores empleados para obtener energía eléctrica}

OPT (Ocean Power Technologies): La instalación de la planta tiene lugar en Escocia, España, Santander. Tiene una potencia de generación de $150 \mathrm{KW}$. El funcionamiento de esta planta undimotriz es a través de sistemas hidráulicos, turbinas hidráulicas, véase la Figura $8[7]$.

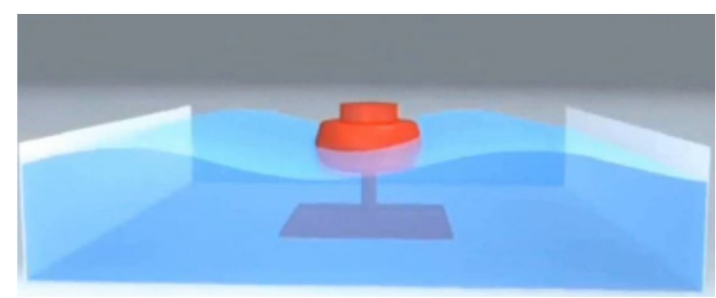

Figura 8. Planta undimotriz OPT. [7]

MUTRIKU Sistemas de columna de agua oscilante OWS: Su implementación se la realizó en España, en la región vasca con fecha de inicio el 8 de julio de 2011. Tiene una potencia de generación de $269 K W$. El funcionamiento de esta planta undimotriz es a través de una cámara la cual genera aire a presión y produce movimiento de turbinas. Puede abastecer a 120 familias. El costo de estas plantas undimotrices es de $6,7 M M$, véase la Figura $9[7]$.

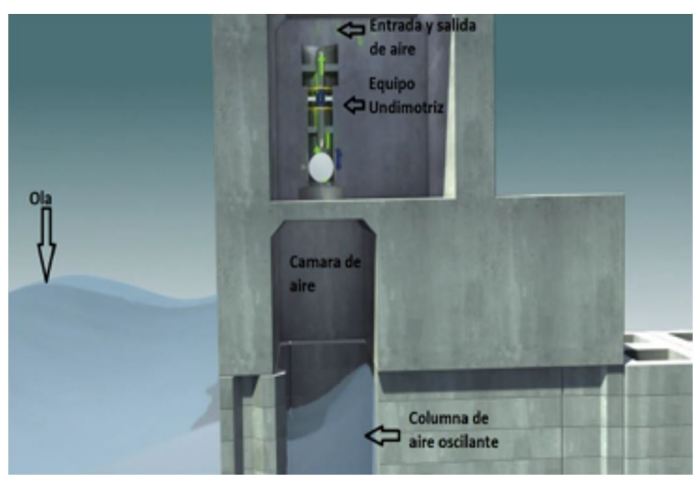

Figura 9. Sistemas de columna de agua oscilante. [7]

PELAMIS: La instalación de la planta tiene lugar en Portugal. Tiene una potencia de generación 
de $750 \mathrm{KW}$. El funcionamiento de esta planta undimotriz es a través de unas boyas en cadena. La transformación a través de un sistema hidráulico, es decir, un movimiento de turbinas hidráulicas, véase la Figura 10 [7].

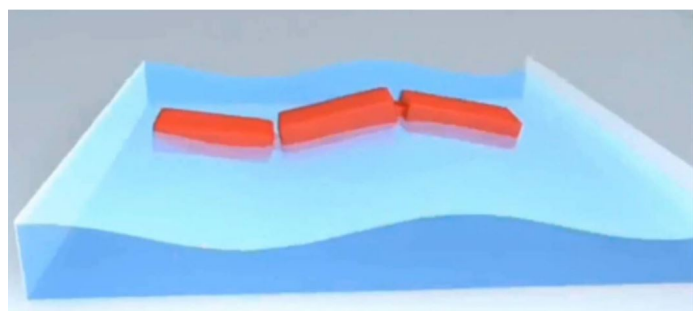

Figura 10. Planta undimotriz PELAMIS. [7]

\section{Conclusiones}

Según los estudios y cálculos realizados sobre el potencial energético del mar ecuatoriano, se llegó a la conclusión que tiene un gran potencial. Se tendría que realizar un estudio de mercado y analizar su costo beneficio para conocer si es conveniente o no su aplicación. Según los cálculos realizados la potencia por metro de frente de ola promedio en el Ecuador es 90,31 KW, aunque esta distribución dista mucho de ser igualitaria, ya que las costas existentes en el Ecuador tienden a tener diferentes potenciales. Aun así se puede concluir que es un potencial bastante bueno. Según el español Víctor Comas, los países con mayor potencial en sus mares son Australia y Nueva Zelanda, alcanzando muchas veces potenciales en sus mares que alcanza los $100 \mathrm{KW}$ por metro de frente de ola. Es por eso por lo que se puede decir que nuestro país tiene un excelente potencial de ola.

Existen diferentes tecnologías para extraer la energía undimotriz y convertirla en eléctrica. Se debe considerar qué convertidor es conveniente a partir de los datos obtenidos, analizando sus ventajas y desventajas.

Uno de los factores negativos es el costo de instalación de una planta undimotriz, ya que para la fabricación de esta planta se tendría que utilizar materiales que soporten el efecto corrosivo del agua salada y vegetación marina sabiendo que estos materiales son más costosos que los usados generalmente.

A pesar de los costos elevados de su implementación, creemos que sería muy importante su aplicación. En nuestras mentes está la frase de David Michael Ross que nos dice: «la energía de las olas no fue diseñada para ahorrar dinero, sino para salvar al mundo».

\section{Referencias}

[1] R. Calero Mendoza and D. Viteri Rivera, "Energía undimotriz alternativa para la producción de energía eléctrica en la Provincia de Santa Elena." Facultad de Ingeniería Industrial, Universidad Estatal Península de Santa Elena (UPSE) Campus la Libertad, 2015.

[2] P. Roldán Chiriboga, "Evaluación de las energías renovables no convencionales factibles de desarrollarse en el Ecuador." Facultad de Ingeniería Eléctrica y Electrónica, Escuela Politécnica Nacional, 2009.

[3] Instituto Nacional de Preinversión, INP, "Estudio de la energía de las olas, corrientes y energía cinética de ríos en el Ecuador," 2013.

[4] LATin. (2014) Introducción al estudio de fuentes renovables de energía. [Online]. Available: http://escritura.proyectolatin.org/ introduccion-al-estudio-de-fuentes-renovables-de\ -energia/prologo-e-introduccion/

[5] Instituto Oceanográfico de la Armada del Ecuador, INOCAR. (2005) Condiciones de olas y aguajes. [Online]. Available: http://www.inocar. mil.ec/web/index.php/boletines/oleajeyaguaje/ 568condiciones-de-olas-y-aguaje-del-8-al-11-de\ -abril-2016.

[6] J. Fernández Chozas, "Energía undimotriz: Una aproximación al aprovechamiento de la energía de las olas para la generación de electricidad," Lambert Academic Publishing, 2012.

[7] M. Pelissero, P. A. Haim, G. Oliveto, F. Galia, and R. Tula, "Aprovechamiento de la energía undimotriz." Universidad Tecnológica Nacional, Facultad Regional de Buenos Aires., 2011.

[8] L. H. Holthuijsen, Waves in Oceanic and Coastal Water, N. York, Ed. Cambridge University Press, 2007.

[9] Instituto Oceanográfico de la Armada del Ecuador (INOCAR). (2001) Informe técnico de tsunami. [Online]. Available: http://www.inocar.mil.ec/web/index.php/ tabla-de-mareas/36-informes-tecnicos.

[10] C. Pastén, "Chile, energía y desarrollo," Obras y proyectos, Scielo, Chile, pp. 28-39, 2012.

[11] R. Zalba. (2013) Consultoría y educación (las olas). [Online]. Available: http://ceambientalblog. blogspot.com/2013/08/las-olas. Html.

[12] ARCONEL. (2013) Plan maestro de electrificación 2013 - 2022. [Online]. Available: http://www.regulacionelectrica.gob.ec/ wp-content/uploads/downloads/2015/12/Vol4 \ -Aspectos-de-sustentabilidad-y-sostenibilidad- $\backslash$ social-y-ambiental.pdf 